\title{
Solvability of some integro-differential equations with anomalous diffusion and transport
}

\author{
Vitali Vougalter $^{1}\left(\mathbb{D} \cdot\right.$ Vitaly Volpert $^{2,3,4}$
}

Received: 15 January 2021 / Revised: 20 June 2021 / Accepted: 22 June 2021

(c) The Author(s), under exclusive licence to Springer Nature Switzerland AG 2021

\begin{abstract}
The article deals with the existence of solutions of an integro-differential equation in the case of anomalous diffusion with the negative Laplace operator in a fractional power in the presence of the transport term. The proof of existence of solutions is based on a fixed point technique. Solvability conditions for elliptic operators without the Fredholm property in unbounded domains are used. We discuss how the introduction of the transport term impacts the regularity of solutions.
\end{abstract}

Keywords Integro-differential equations · Non Fredholm operators · Sobolev spaces

Mathematics Subject Classification 35R11 · 35K57 · 35R09

\section{Introduction}

The present article is devoted to the existence of stationary solutions of the following nonlocal reaction-diffusion equation for $0<s<\frac{1}{4}$ and the nontrivial constant $b \in \mathbb{R}$

$$
\frac{\partial u}{\partial t}=-D\left(-\frac{\partial^{2}}{\partial x^{2}}\right)^{s} u+b \frac{\partial u}{\partial x}+\int_{-\infty}^{\infty} K(x-y) g(u(y, t)) d y+f(x),
$$

\footnotetext{
$凶 \quad$ Vitali Vougalter

vitali@math.toronto.edu

Vitaly Volpert

volpert@math.univ-lyon1.fr

1 Department of Mathematics, University of Toronto, Toronto, ON M5S 2E4, Canada

2 UMR 5208 CNRS, Institute Camille Jordan, University Lyon 1, 69622 Villeurbanne, France

3 INRIA Team Dracula, INRIA Lyon La Doua, 69603 Villeurbanne, France

4 Peoples' Friendship University of Russia (RUDN University), 6 Miklukho-Maklaya St, Moscow, Russian Federation 117198
} 
which appears in the cell population dynamics. Note that the solvability of the equation analogous to (1.1) without the transport term was addressed in [36]. Emergence and propagation of patterns in nonlocal reaction-diffusion equations arising in the theory of speciation and containing the drift term were discussed in [26]. The space variable $x$ here corresponds to the cell genotype, $u(x, t)$ denotes the cell density as a function of their genotype and time. The right side of this equation describes the evolution of cell density via cell proliferation, mutations, transport and cell influx/efflux. The anomalous diffusion term here corresponds to the change of genotype due to small random mutations, and the integral term describes large mutations. Function $g(u)$ stands for the rate of cell birth which depends on $u$ (density dependent proliferation), and the kernel $K(x-y)$ gives the proportion of newly born cells changing their genotype from $y$ to $x$. Let us assume that it depends on the distance between the genotypes. Finally, the last term in the right side of this problem designates the influx/efflux of cells for different genotypes.

The operator $\left(-\frac{\partial^{2}}{\partial x^{2}}\right)^{s}$ in Eq. (1.1) describes a particular case of the anomalous diffusion actively studied in the context of different applications in: plasma physics and turbulence [8,23], surface diffusion [17,21], semiconductors [22] and so on. It is largely used in the works on the nonlocal diffusive processes. The probabilistic realization of the anomalous diffusion was discussed in [20]. The operator $\left(-\frac{\partial^{2}}{\partial x^{2}}\right)^{s}$ is defined by means of the spectral calculus. This is the pseudo-differential operator with symbol $|p|^{2 s}$, namely

$$
\left(-\frac{d^{2}}{d x^{2}}\right)^{s} \phi(x)=\frac{1}{\sqrt{2 \pi}} \int_{-\infty}^{\infty}|p|^{2 s} \widehat{\phi}(p) e^{i p x} d p, \quad \phi(x) \in H^{2 s}(\mathbb{R})
$$

with the standard Fourier transform defined in (2.1). In the present work we will consider the case of $0<s<1 / 4$.

Let us set $D=1$ and establish the existence of solutions of the problem

$$
-\left(-\frac{d^{2}}{d x^{2}}\right)^{s} u+b \frac{d u}{d x}+\int_{-\infty}^{\infty} K(x-y) g(u(y)) d y+f(x)=0
$$

with $0<s<\frac{1}{4}$, considering the case where the linear part of this operator fails to satisfy the Fredholm property. As a consequence, the conventional methods of the nonlinear analysis may not be applicable. We use the solvability conditions for non Fredholm operators along with the method of the contraction mappings. Equation (1.2) is the stationary case of problem (1.1), and we solve it using (1.8), (1.9) and (1.11). Since our model describes the distribution of a population density with respect to the genotype, the existence of stationary solutions of (1.1) corresponds to the existence of biological species.

To introduce the concept of the non Fredholm operators, we consider the equation

$$
-\Delta u+V(x) u-a u=f,
$$


where $u \in E=H^{2}\left(\mathbb{R}^{d}\right)$ and $f \in F=L^{2}\left(\mathbb{R}^{d}\right), d \in \mathbb{N}, a$ is a constant and the scalar potential function $V(x)$ is either zero identically or tends to 0 at infinity. If $a \geq 0$, the origin belongs to the essential spectrum of the operator $A: E \rightarrow F$ corresponding to the left side of problem (1.3). Consequently, such an operator fails to satisfy the Fredholm property. Its image is not closed, for $d>1$ the dimension of its kernel and the codimension of its image are not finite. The present work is devoted to the studies of certain properties of the operators of this kind. Note that for the Fredholm property we consider bounded but not $H^{2}\left(\mathbb{R}^{d}\right)$ solutions of the corresponding homogeneous adjoint problem. If $V(x) \equiv 0$, Eq. (1.3) has constant coefficients and we can apply the Fourier transform to solve it explicitly. If $f \in L^{2}\left(\mathbb{R}^{d}\right)$ and $x f \in L^{1}\left(\mathbb{R}^{d}\right)$, then it has a unique solution in $H^{2}\left(\mathbb{R}^{d}\right)$ if and only if

$$
\left(f(x), \frac{e^{i p x}}{(2 \pi)^{\frac{d}{2}}}\right)_{L^{2}\left(\mathbb{R}^{d}\right)}=0, \quad p \in S_{\sqrt{a}}^{d} \text { a.e. }
$$

(see Lemmas 5 and 6 of [35]). Here $S_{\sqrt{a}}^{d}$ stands for the sphere in $\mathbb{R}^{d}$ of radius $\sqrt{a}$ centered at the origin. Hence, though our operator fails to satisfy the Fredholm property, the solvability relations are formulated similarly. However, this similarity is only formal since the range of the operator is not closed. The orthogonality conditions (1.4) are with respect to the standard Fourier harmonics, which solve the homogeneous adjoint problem for (1.3) when the scalar potential function vanishes, belong to $L^{\infty}\left(\mathbb{R}^{d}\right)$ but they are not square integrable.

Note that elliptic problems with non Fredholm operators were studied actively in recent years. Approaches in weighted Sobolev and Hölder spaces were developed in [3-7]. In particular, when $a=0$ the operator $A$ is Fredholm in some properly chosen weighted spaces (see [3-7]). However, the case of $a \neq 0$ is considerably different and the method developed in these articles is not applicable. The non Fredholm Schrödinger type operators were treated with the methods of the spectral and the scattering theory in $[14,24,31]$. The Laplace operator with drift from the point of view of non Fredholm operators was considered in [33] and linearized Cahn-Hilliard problems in [25,34]. Fredholm structures, topological invariants and applications were covered in [12]. Fredholm and properness properties of quasilinear elliptic systems of second order were discussed in [15]. Nonlinear non Fredholm elliptic equations were studied in $[13,32,35]$. Important applications to the theory of reaction-diffusion equations were developed in [10,11]. Non Fredholm operators arise also in the context of the wave systems with an infinite number of localized traveling waves (see [1]). Standing lattice solitons in the discrete NLS equation with saturation were studied in [2]. Weak solutions of the Dirichlet and Neumann problems with drift were considered in [18]. Work [19] deals with the imbedding theorems and the spectrum of a certain pseudodifferential operator. Front propagation equations with anomalous diffusion were studied actively in recent years (see e.g. [28,29]).

We set $K(x)=\varepsilon \mathcal{K}(x)$, where $\varepsilon \geq 0$. The small, nonnegative parameter $\varepsilon$ has the biological meaning that the integral production term is small with respect to the others, that is, the frequency of large mutations is sufficiently small. Let us suppose that the assumption below is fulfilled. 
Assumption 1 Consider $0<s<\frac{1}{4}$. The constant $b \in \mathbb{R}, b \neq 0$. Let $f(x): \mathbb{R} \rightarrow \mathbb{R}$ be nontrivial, such that $f(x) \in L^{1}(\mathbb{R}) \cap L^{2}(\mathbb{R})$. Assume also that $\mathcal{K}(x): \mathbb{R} \rightarrow \mathbb{R}$ is nontrivial and $\mathcal{K}(x) \in L^{1}(\mathbb{R}) \cap L^{2}(\mathbb{R})$.

Note that as distinct from Assumption 1.1 of [36] we do not need to assume here that $\left(-\frac{d^{2}}{d x^{2}}\right)^{\frac{1}{2}-s} f(x) \in L^{2}(\mathbb{R})$, which is the advantage of introducing the transport term into our equation. We also do not need to impose the regularity condition $\left(-\frac{d^{2}}{d x^{2}}\right)^{\frac{1}{2}-s} \mathcal{K}(x) \in L^{2}(\mathbb{R})$ on the integral kernel of our problem. From the point of view of applications, the space dimension is not restricted to $d=1$ since the space variable corresponds to the cell genotype but not to the usual physical space. We use the Sobolev spaces

$$
H^{2 s}(\mathbb{R}):=\left\{u(x): \mathbb{R} \rightarrow \mathbb{R} \mid u(x) \in L^{2}(\mathbb{R}),\left(-\frac{d^{2}}{d x^{2}}\right)^{s} u \in L^{2}(\mathbb{R})\right\}, \quad 0<s \leq 1
$$

equipped with the norm

$$
\|u\|_{H^{2 s}(\mathbb{R})}^{2}:=\|u\|_{L^{2}(\mathbb{R})}^{2}+\left\|\left(-\frac{d^{2}}{d x^{2}}\right)^{s} u\right\|_{L^{2}(\mathbb{R})}^{2} .
$$

Evidently, in the particular case of $s=\frac{1}{2}$ we have

$$
\|u\|_{H^{1}(\mathbb{R})}^{2}:=\|u\|_{L^{2}(\mathbb{R})}^{2}+\left\|\frac{d u}{d x}\right\|_{L^{2}(\mathbb{R})}^{2} .
$$

The standard Sobolev inequality in one dimension (see e.g. Section 8.5 of [16]) yields

$$
\|u\|_{L^{\infty}(\mathbb{R})} \leq \frac{1}{\sqrt{2}}\|u\|_{H^{1}(\mathbb{R})} .
$$

When our nonnegative parameter $\varepsilon=0$, we obtain the linear equation (4.1) with $a=0$ and $0<s<\frac{1}{4}$. By virtue of assertion (3) of Lemma 6 below along with Assumption 1 in this case Eq. (4.1) possesses a unique solution

$$
u_{0}(x) \in H^{1}(\mathbb{R}), \quad 0<s<\frac{1}{4},
$$

so that no orthogonality conditions are required. According to assertions (4) and (5) of Lemma 6, when $a=0$, a certain orthogonality relation (4.5) is needed to be able to solve problem (4.1) in $H^{1}(\mathbb{R})$ for $\frac{1}{4} \leq s \leq \frac{1}{2}$ and in $H^{2 s}(\mathbb{R})$ if $\frac{1}{2}<s<1$. Clearly, 
$u_{0}(x)$ does not vanish identically on the real line since our influx/efflux term $f(x)$ is nontrivial as assumed.

Note that in the analogous situation in the absence of the transport term discussed in [36] the corresponding Poisson type equation with the negative Laplacian raised to a fractional power admits a unique solution

$$
u_{0}(x) \in H^{2 s}(\mathbb{R}), \quad 0<s<\frac{1}{4}
$$

which belongs to $H^{1}(\mathbb{R})$ under the extra regularity assumption on the influx/efflux term. The inequality $0<s<\frac{1}{4}$ is also needed to obtain the finite upper bounds on the integral terms throughout the article.

Let us look for the resulting solution of nonlinear problem (1.2) as

$$
u(x)=u_{0}(x)+u_{p}(x)
$$

Obviously, we arrive at the perturbative equation

$$
\left(-\frac{d^{2}}{d x^{2}}\right)^{s} u_{p}-b \frac{d u_{p}}{d x}=\varepsilon \int_{-\infty}^{\infty} \mathcal{K}(x-y) g\left(u_{0}(y)+u_{p}(y)\right) d y, \quad 0<s<\frac{1}{4} \text {. }
$$

For technical purposes we introduce a closed ball in the Sobolev space

$$
B_{\rho}:=\left\{u(x) \in H^{1}(\mathbb{R}) \mid\|u\|_{H^{1}(\mathbb{R})} \leq \rho\right\}, \quad 0<\rho \leq 1 .
$$

Let us seek the solution of Eq. (1.9) as the fixed point of the auxiliary nonlinear problem

$$
\left(-\frac{d^{2}}{d x^{2}}\right)^{s} u-b \frac{d u}{d x}=\varepsilon \int_{-\infty}^{\infty} \mathcal{K}(x-y) g\left(u_{0}(y)+v(y)\right) d y, \quad 0<s<\frac{1}{4}
$$

in the ball (1.10). For a given function $v(y)$ this is an equation to be solved for $u(x)$. The left side of (1.11) contains the non Fredholm operator

$$
L_{0, b, s}: H^{1}(\mathbb{R}) \rightarrow L^{2}(\mathbb{R}), \quad 0<s<\frac{1}{4},
$$

defined in (4.2) which has no bounded inverse. The spectral properties of such operator are discussed in the final section of the work. A similar situation appeared in earlier articles [32,35] but as distinct from the present case, the problems discussed there required orthogonality relations. The fixed point technique was used in [30] to evaluate the perturbation to the standing solitary wave of the Nonlinear Schrödinger (NLS) equation when either the external potential or the nonlinear term in the NLS 
were perturbed but the Schrödinger operator involved in the nonlinear problem there possessed the Fredholm property (see Assumption 1 of [30], also [9]). For the technical purposes we introduce the interval on the real line

$$
I:=\left[-\frac{1}{\sqrt{2}}\left\|u_{0}\right\|_{H^{1}(\mathbb{R})}-\frac{1}{\sqrt{2}}, \frac{1}{\sqrt{2}}\left\|u_{0}\right\|_{H^{1}(\mathbb{R})}+\frac{1}{\sqrt{2}}\right]
$$

along with the closed ball in the space of $C_{2}(I)$ functions, namely

$$
D_{M}:=\left\{g(z) \in C_{2}(I) \mid\|g\|_{C_{2}(I)} \leq M\right\}, \quad M>0 .
$$

We will use the norm

$$
\|g\|_{C_{2}(I)}:=\|g\|_{C(I)}+\left\|g^{\prime}\right\|_{C(I)}+\left\|g^{\prime \prime}\right\|_{C(I)},
$$

where $\|g\|_{C(I)}:=\max _{z \in I}|g(z)|$. Let us make the following assumption on the nonlinear part of problem (1.2).

Assumption 2 Let $g(z): \mathbb{R} \rightarrow \mathbb{R}$, such that $g(0)=0$ and $g^{\prime}(0)=0$. In addition to that $g(z) \in D_{M}$ and it does not vanish identically on the interval $I$.

Let us explain why we impose the condition $g^{\prime}(0)=0$. If $g^{\prime}(0) \neq 0$ and the Fourier image of our integral kernel does not vanish at zero, then the essential spectrum of the corresponding linearized operator does not contain the origin. The operator satisfies the Fredholm property, and the conventional methods of the nonlinear analysis are applicable here. If $g^{\prime}(0)=0$, then the operator fails to satisfy the Fredholm property, and the goal of this article is to establish the existence of solutions in such case where usual methods are not applicable. Thus we impose this condition on the nonlinearity. From the point of view of the applications to the Mathematical Biology, it means that we keep in mind, for instance the quadratic function $g(z)$, such that the cell proliferation rate depends on the cell-cell interaction.

Let us introduce the operator $T_{g}$, such that $u=T_{g} v$, where $u$ is a solution of problem (1.11). Our first main result is as follows.

Theorem 3 Let Assumptions 1 and 2 hold. Then problem (1.11) defines the map $T_{g}: B_{\rho} \rightarrow B_{\rho}$, which is a strict contraction for all

$$
\begin{aligned}
0 & <\varepsilon \leq \frac{\rho}{2 M\left(\left\|u_{0}\right\|_{H^{1}(\mathbb{R})}+1\right)^{2}} \\
& \times\left\{\frac{\|\mathcal{K}\|_{L^{1}(\mathbb{R})}^{2}\left(\left\|u_{0}\right\|_{H^{1}(\mathbb{R})}+1\right)^{8 s-2}}{(1-4 s)(16 \pi s)^{4 s}}+\frac{\|\mathcal{K}\|_{L^{2}(\mathbb{R})}^{2}}{4 b^{2}}\right\}^{-\frac{1}{2}} .
\end{aligned}
$$

The unique fixed point $u_{p}(x)$ of this map $T_{g}$ is the only solution of Eq. (1.9) in $B_{\rho}$. 
Note that condition (1.15) can be easily reformulated in terms of the original kernel $K$ appearing in our Eq. (1.2), namely

$$
4 M^{2}\left(\left\|u_{0}\right\|_{H^{1}(\mathbb{R})}+1\right)^{4}\left\{\frac{\|K\|_{L^{1}(\mathbb{R})}^{2}\left(\left\|u_{0}\right\|_{H^{1}(\mathbb{R})}+1\right)^{8 s-2}}{(1-4 s)(16 \pi s)^{4 s}}+\frac{\|K\|_{L^{2}(\mathbb{R})}^{2}}{4 b^{2}}\right\} \leq \rho^{2}
$$

for some $\rho \in(0,1]$. Evidently, the cumulative solution of problem (1.2) given by (1.8) will be nontrivial since the influx/efflux term $f(x)$ is nontrivial and $g(0)$ vanishes as assumed. Let us make use of the following elementary lemma.

Lemma 4 For $R \in(0,+\infty)$ consider the function

$$
\varphi(R):=\alpha R^{1-4 s}+\frac{\beta}{R^{4 s}}, \quad 0<s<\frac{1}{4}, \quad \alpha, \beta>0 .
$$

It achieves the minimal value at $R^{*}:=\frac{4 \beta s}{\alpha(1-4 s)}$, which is given by

$$
\varphi\left(R^{*}\right)=\frac{(1-4 s)^{4 s-1}}{(4 s)^{4 s}} \alpha^{4 s} \beta^{1-4 s}
$$

Our second main result is about the continuity of the resulting solution of Eq. (1.2) given by (1.8) with respect to the nonlinear function $g$. The continuity of such solution with respect to the transport parameter $b$ will be studied in our consecutive work. Let us introduce the following positive, auxiliary expression

$$
\sigma:=M\left(\left\|u_{0}\right\|_{H^{1}(\mathbb{R})}+1\right)\left\{\frac{\|\mathcal{K}\|_{L^{1}(\mathbb{R})}^{2}\left(\left\|u_{0}\right\|_{H^{1}(\mathbb{R})}+1\right)^{8 s-2}}{(1-4 s)(4 \pi s)^{4 s}}+\frac{\|\mathcal{K}\|_{L^{2}(\mathbb{R})}^{2}}{b^{2}}\right\}^{\frac{1}{2}} .
$$

Theorem 5 Let $j=1,2$, the assumptions of Theorem 3 including inequality (1.15) are valid, such that $u_{p, j}(x)$ is the unique fixed point of the map $T_{g_{j}}: B_{\rho} \rightarrow B_{\rho}$, which is a strict contraction for all the values of $\varepsilon$ satisfying (1.15) and the cumulative solution of Eq. (1.2) with $g(z)=g_{j}(z)$ is given by

$$
u_{j}(x):=u_{0}(x)+u_{p, j}(x) .
$$

Then for all $\varepsilon$, which satisfy estimate (1.15) the upper bound

$$
\begin{aligned}
& \left\|u_{1}(x)-u_{2}(x)\right\|_{H^{1}(\mathbb{R})} \leq \frac{\varepsilon}{1-\varepsilon \sigma}\left(\left\|u_{0}\right\|_{H^{1}(\mathbb{R})}+1\right)^{2} \\
& \times\left[\frac{\|\mathcal{K}\|_{L^{1}(\mathbb{R})}^{2}\left(\left\|u_{0}\right\|_{H^{1}(\mathbb{R})}+1\right)^{8 s-2}}{(1-4 s)(16 \pi s)^{4 s}}+\frac{\|\mathcal{K}\|_{L^{2}(\mathbb{R})}^{2}}{4 b^{2}}\right]^{\frac{1}{2}}\left\|g_{1}-g_{2}\right\|_{C_{2}(I)}
\end{aligned}
$$

holds. 
Let us proceed to the proof of our first main statement.

\section{The existence of the perturbed solution}

Proof of Theorem 3 We choose an arbitrary $v(x) \in B_{\rho}$ and designate the term involved in the integral expression in the right side of Eq. (1.11) as

$$
G(x):=g\left(u_{0}(x)+v(x)\right) .
$$

Throughout the article we will use the standard Fourier transform

$$
\widehat{\phi}(p):=\frac{1}{\sqrt{2 \pi}} \int_{-\infty}^{\infty} \phi(x) e^{-i p x} d x .
$$

Evidently, we have the inequality

$$
\|\widehat{\phi}(p)\|_{L^{\infty}(\mathbb{R})} \leq \frac{1}{\sqrt{2 \pi}}\|\phi(x)\|_{L^{1}(\mathbb{R})} .
$$

Let us apply (2.1) to both sides of Eq. (1.11). This yields

$$
\widehat{u}(p)=\varepsilon \sqrt{2 \pi} \frac{\widehat{\mathcal{K}}(p) \widehat{G}(p)}{|p|^{2 s}-i b p} .
$$

Then for the norm we arrive at

$$
\|u\|_{L^{2}(\mathbb{R})}^{2}=2 \pi \varepsilon^{2} \int_{-\infty}^{\infty} \frac{|\widehat{\mathcal{K}}(p)|^{2}|\widehat{G}(p)|^{2}}{|p|^{4 s}+b^{2} p^{2}} d p \leq 2 \pi \varepsilon^{2} \int_{-\infty}^{\infty} \frac{|\widehat{\mathcal{K}}(p)|^{2}|\widehat{G}(p)|^{2}}{|p|^{4 s}} d p .
$$

As distinct from articles [32,35] involving the standard Laplacian in the diffusion term, here we do not try to control the norm

$$
\left\|\frac{\widehat{\mathcal{K}}(p)}{|p|^{2 s}}\right\|_{L^{\infty}(\mathbb{R})}
$$

Instead, we estimate the right side of (2.4) using the analog of bound (2.2) applied to functions $\mathcal{K}$ and $G$ with $R \in(0,+\infty)$ as

$$
\begin{aligned}
& 2 \pi \varepsilon^{2} \int_{|p| \leq R} \frac{|\widehat{\mathcal{K}}(p)|^{2}|\widehat{G}(p)|^{2}}{|p|^{4 s}} d p+2 \pi \varepsilon^{2} \int_{|p|>R} \frac{|\widehat{\mathcal{K}}(p)|^{2}|\widehat{G}(p)|^{2}}{|p|^{4 s}} d p \\
& \quad \leq \varepsilon^{2}\|\mathcal{K}\|_{L^{1}(\mathbb{R})}^{2}\left\{\frac{1}{\pi}\|G(x)\|_{L^{1}(\mathbb{R})}^{2} \frac{R^{1-4 s}}{1-4 s}+\frac{1}{R^{4 s}}\|G(x)\|_{L^{2}(\mathbb{R})}^{2}\right\} .
\end{aligned}
$$


Because $v(x) \in B_{\rho}$, we have

$$
\left\|u_{0}+v\right\|_{L^{2}(\mathbb{R})} \leq\left\|u_{0}\right\|_{H^{1}(\mathbb{R})}+1 .
$$

Sobolev inequality (1.7) gives us

$$
\left\|u_{0}+v\right\|_{L^{\infty}(\mathbb{R})} \leq \frac{1}{\sqrt{2}}\left(\left\|u_{0}\right\|_{H^{1}(\mathbb{R})}+1\right) .
$$

Let us use the formula

$$
G(x)=\int_{0}^{u_{0}+v} g^{\prime}(z) d z
$$

Hence

$$
|G(x)| \leq \max _{z \in I}\left|g^{\prime}(z)\right|\left|u_{0}+v\right| \leq M\left|u_{0}+v\right|,
$$

where the interval $I$ is defined in (1.12). Then

$$
\|G(x)\|_{L^{2}(\mathbb{R})} \leq M\left\|u_{0}+v\right\|_{L^{2}(\mathbb{R})} \leq M\left(\left\|u_{0}\right\|_{H^{1}(\mathbb{R})}+1\right) .
$$

Since

$$
G(x)=\int_{0}^{u_{0}+v} d y\left[\int_{0}^{y} g^{\prime \prime}(z) d z\right]
$$

we derive

$$
|G(x)| \leq \frac{1}{2} \max _{z \in I}\left|g^{\prime \prime}(z)\right|\left|u_{0}+v\right|^{2} \leq \frac{M}{2}\left|u_{0}+v\right|^{2}
$$

so that

$$
\|G(x)\|_{L^{1}(\mathbb{R})} \leq \frac{M}{2}\left\|u_{0}+v\right\|_{L^{2}(\mathbb{R})}^{2} \leq \frac{M}{2}\left(\left\|u_{0}\right\|_{H^{1}(\mathbb{R})}+1\right)^{2} .
$$

Therefore, we arrive at the upper bound for the right side of (2.5) given by

$$
\varepsilon^{2}\|\mathcal{K}\|_{L^{1}(\mathbb{R})}^{2} M^{2}\left(\left\|u_{0}\right\|_{H^{1}(\mathbb{R})}+1\right)^{2}\left\{\frac{\left(\left\|u_{0}\right\|_{H^{1}(\mathbb{R})}+1\right)^{2} R^{1-4 s}}{4 \pi(1-4 s)}+\frac{1}{R^{4 s}}\right\},
$$

with $R \in(0,+\infty)$. By virtue of Lemma 4 we evaluate the minimal value of the expression above. Therefore,

$$
\|u\|_{L^{2}(\mathbb{R})}^{2} \leq \varepsilon^{2}\|\mathcal{K}\|_{L^{1}(\mathbb{R})}^{2}\left(\left\|u_{0}\right\|_{H^{1}(\mathbb{R})}+1\right)^{2+8 s} \frac{M^{2}}{(1-4 s)(16 \pi s)^{4 s}} .
$$


Using (2.3) we obtain

$$
\int_{-\infty}^{\infty} p^{2}|\widehat{u}(p)|^{2} d p \leq \frac{2 \pi \varepsilon^{2}}{b^{2}} \int_{-\infty}^{\infty}|\widehat{\mathcal{K}}(p)|^{2}|\widehat{G}(p)|^{2} d p
$$

By means of the analog of inequality (2.2) applied to function $G$ along with bound (2.6) we derive

$$
\left\|\frac{d u}{d x}\right\|_{L^{2}(\mathbb{R})}^{2} \leq \frac{\varepsilon^{2}}{b^{2}}\|G\|_{L^{1}(\mathbb{R})}^{2}\|\mathcal{K}\|_{L^{2}(\mathbb{R})}^{2} \leq \frac{\varepsilon^{2} M^{2}}{4 b^{2}}\left(\left\|u_{0}\right\|_{H^{1}(\mathbb{R})}+1\right)^{4}\|\mathcal{K}\|_{L^{2}(\mathbb{R})}^{2} .
$$

Let us apply the definition of the norm (1.6) along with inequalities (2.7) and (2.8) to arrive at the estimate from above for $\|u\|_{H^{1}(\mathbb{R})}$ given by

$$
\varepsilon\left(\left\|u_{0}\right\|_{H^{1}(\mathbb{R})}+1\right)^{2} M\left[\frac{\|\mathcal{K}\|_{L^{1}(\mathbb{R})}^{2}\left(\left\|u_{0}\right\|_{H^{1}(\mathbb{R})}+1\right)^{8 s-2}}{(1-4 s)(16 \pi s)^{4 s}}+\frac{\|\mathcal{K}\|_{L^{2}(\mathbb{R})}^{2}}{4 b^{2}}\right]^{\frac{1}{2}} \leq \frac{\rho}{2}
$$

for all values of the parameter $\varepsilon$ satisfying inequality (1.15), so that $u(x) \in B_{\rho}$ as well. Let us suppose that for a certain $v(x) \in B_{\rho}$ there exist two solutions $u_{1,2}(x) \in B_{\rho}$ of Eq. (1.11). Then their difference $w(x):=u_{1}(x)-u_{2}(x) \in H^{1}(\mathbb{R})$ solves

$$
\left(-\frac{d^{2}}{d x^{2}}\right)^{s} w-b \frac{d w}{d x}=0, \quad 0<s<\frac{1}{4}
$$

Clearly, the operator $L_{0, b, s}: H^{1}(\mathbb{R}) \rightarrow L^{2}(\mathbb{R})$ in the left side of (2.10) defined in (4.2) does not have any nontrivial zero modes, such that $w(x) \equiv 0$ on the real line. Thus, Eq. (1.11) defines a map $T_{g}: B_{\rho} \rightarrow B_{\rho}$ for all $\varepsilon$ satisfying bound (1.15).

Let us demonstrate that this map is a strict contraction. We choose arbitrarily $v_{1,2}(x) \in B_{\rho}$. By virtue of the argument above $u_{1,2}:=T_{g} v_{1,2} \in B_{\rho}$ as well when $\varepsilon$ satisfies (1.15). According to (1.11) we have

$$
\begin{aligned}
& \left(-\frac{d^{2}}{d x^{2}}\right)^{s} u_{1}-b \frac{d u_{1}}{d x}=\varepsilon \int_{-\infty}^{\infty} \mathcal{K}(x-y) g\left(u_{0}(y)+v_{1}(y)\right) d y, \\
& \left(-\frac{d^{2}}{d x^{2}}\right)^{s} u_{2}-b \frac{d u_{2}}{d x}=\varepsilon \int_{-\infty}^{\infty} \mathcal{K}(x-y) g\left(u_{0}(y)+v_{2}(y)\right) d y
\end{aligned}
$$

with $0<s<\frac{1}{4}$. Let us define

$$
G_{1}(x):=g\left(u_{0}(x)+v_{1}(x)\right), \quad G_{2}(x):=g\left(u_{0}(x)+v_{2}(x)\right)
$$


and apply the standard Fourier transform (2.1) to both sides of Eqs. (2.11) and (2.12). This yields

$$
\widehat{u_{1}}(p)=\varepsilon \sqrt{2 \pi} \frac{\widehat{\mathcal{K}}(p) \widehat{G_{1}}(p)}{|p|^{2 s}-i b p}, \quad \widehat{u_{2}}(p)=\varepsilon \sqrt{2 \pi} \frac{\widehat{\mathcal{K}}(p) \widehat{G_{2}}(p)}{|p|^{2 s}-i b p}
$$

Obviously,

$$
\begin{aligned}
& \left\|u_{1}-u_{2}\right\|_{L^{2}(\mathbb{R})}^{2}=\varepsilon^{2} 2 \pi \int_{-\infty}^{\infty} \frac{|\widehat{\mathcal{K}}(p)|^{2}\left|\widehat{G_{1}}(p)-\widehat{G_{2}}(p)\right|^{2}}{|p|^{4 s}+b^{2} p^{2}} d p \\
& \leq \varepsilon^{2} 2 \pi \int_{-\infty}^{\infty} \frac{|\widehat{\mathcal{K}}(p)|^{2}\left|\widehat{G_{1}}(p)-\widehat{G_{2}}(p)\right|^{2}}{|p|^{4 s}} d p .
\end{aligned}
$$

Clearly, the right side of (2.14) can be estimated from above via inequality (2.2) as

$$
\begin{aligned}
& \varepsilon^{2} 2 \pi\left[\int_{|p| \leq R} \frac{|\widehat{\mathcal{K}}(p)|^{2}\left|\widehat{G_{1}}(p)-\widehat{G_{2}}(p)\right|^{2}}{|p|^{4 s}} d p+\int_{|p|>R} \frac{|\widehat{\mathcal{K}}(p)|^{2}\left|\widehat{G_{1}}(p)-\widehat{G_{2}}(p)\right|^{2}}{|p|^{4 s}} d p\right] \\
& \leq \varepsilon^{2}\|\mathcal{K}\|_{L^{1}(\mathbb{R})}^{2}\left\{\frac{1}{\pi}\left\|G_{1}(x)-G_{2}(x)\right\|_{L^{1}(\mathbb{R})}^{2} \frac{R^{1-4 s}}{1-4 s}+\left\|G_{1}(x)-G_{2}(x)\right\|_{L^{2}(\mathbb{R})}^{2} \frac{1}{R^{4 s}}\right\},
\end{aligned}
$$

where $R \in(0,+\infty)$. We express

$$
G_{1}(x)-G_{2}(x)=\int_{u_{0}+v_{2}}^{u_{0}+v_{1}} g^{\prime}(z) d z
$$

Hence

$$
\left|G_{1}(x)-G_{2}(x)\right| \leq \max _{z \in I}\left|g^{\prime}(z)\right|\left|v_{1}-v_{2}\right| \leq M\left|v_{1}-v_{2}\right|
$$

so that

$$
\left\|G_{1}(x)-G_{2}(x)\right\|_{L^{2}(\mathbb{R})} \leq M\left\|v_{1}-v_{2}\right\|_{L^{2}(\mathbb{R})} \leq M\left\|v_{1}-v_{2}\right\|_{H^{1}(\mathbb{R})} .
$$

Evidently,

$$
G_{1}(x)-G_{2}(x)=\int_{u_{0}+v_{2}}^{u_{0}+v_{1}} d y\left[\int_{0}^{y} g^{\prime \prime}(z) d z\right]
$$

This enables us to obtain an upper bound for the absolute value of $G_{1}(x)-G_{2}(x)$ as

$$
\frac{1}{2} \max _{z \in I}\left|g^{\prime \prime}(z)\right|\left|\left(v_{1}-v_{2}\right)\left(2 u_{0}+v_{1}+v_{2}\right)\right| \leq \frac{M}{2}\left|\left(v_{1}-v_{2}\right)\left(2 u_{0}+v_{1}+v_{2}\right)\right|
$$


The Schwarz inequality gives us the estimate from above for the norm $\| G_{1}(x)-$ $G_{2}(x) \|_{L^{1}(\mathbb{R})}$ as

$$
\frac{M}{2}\left\|v_{1}-v_{2}\right\|_{L^{2}(\mathbb{R})}\left\|2 u_{0}+v_{1}+v_{2}\right\|_{L^{2}(\mathbb{R})} \leq M\left\|v_{1}-v_{2}\right\|_{H^{1}(\mathbb{R})}\left(\left\|u_{0}\right\|_{H^{1}(\mathbb{R})}+1\right) .
$$

Thus we arrive at the upper bound for the norm $\left\|u_{1}(x)-u_{2}(x)\right\|_{L^{2}(\mathbb{R})}^{2}$ given by

$$
\varepsilon^{2}\|\mathcal{K}\|_{L^{1}(\mathbb{R})}^{2} M^{2}\left\|v_{1}-v_{2}\right\|_{H^{1}(\mathbb{R})}^{2}\left\{\frac{1}{\pi}\left(\left\|u_{0}\right\|_{H^{1}(\mathbb{R})}+1\right)^{2} \frac{R^{1-4 s}}{1-4 s}+\frac{1}{R^{4 s}}\right\}, \quad 0<s<\frac{1}{4} .
$$

Lemma 4 allows us to minimize the expression above over $R \in(0,+\infty)$. This yields the estimate from above for $\left\|u_{1}(x)-u_{2}(x)\right\|_{L^{2}(\mathbb{R})}^{2}$ as

$$
\varepsilon^{2}\|\mathcal{K}\|_{L^{1}(\mathbb{R})}^{2} M^{2}\left\|v_{1}-v_{2}\right\|_{H^{1}(\mathbb{R})}^{2} \frac{\left(\left\|u_{0}\right\|_{H^{1}(\mathbb{R})}+1\right)^{8 s}}{(1-4 s)(4 \pi s)^{4 s}} .
$$

By virtue of (2.13) we derive

$$
\int_{-\infty}^{\infty} p^{2}\left|\widehat{u}_{1}(p)-\widehat{u}_{2}(p)\right|^{2} d p \leq \frac{2 \pi \varepsilon^{2}}{b^{2}} \int_{-\infty}^{\infty}|\widehat{\mathcal{K}}(p)|^{2}\left|\widehat{G}_{1}(p)-\widehat{G}_{2}(p)\right|^{2} d p .
$$

Inequalities (2.2) and (2.15) imply that

$$
\begin{aligned}
& \left\|\frac{d}{d x}\left(u_{1}-u_{2}\right)\right\|_{L^{2}(\mathbb{R})}^{2} \leq \frac{\varepsilon^{2}}{b^{2}}\|\mathcal{K}\|_{L^{2}(\mathbb{R})}^{2}\left\|G_{1}-G_{2}\right\|_{L^{1}(\mathbb{R})}^{2} \\
& \leq \frac{\varepsilon^{2}}{b^{2}}\|\mathcal{K}\|_{L^{2}(\mathbb{R})}^{2} M^{2}\left\|v_{1}-v_{2}\right\|_{H^{1}(\mathbb{R})}^{2}\left(\left\|u_{0}\right\|_{H^{1}(\mathbb{R})}+1\right)^{2} .
\end{aligned}
$$

According to (2.16) and (2.17) along with definition (1.6) the norm $\left\|u_{1}-u_{2}\right\|_{H^{1}(\mathbb{R})}$ can be bounded from above by the expression

$$
\begin{aligned}
& \varepsilon M\left(\left\|u_{0}\right\|_{H^{1}(\mathbb{R})}+1\right) \\
& \quad \times\left\{\frac{\|\mathcal{K}\|_{L^{1}(\mathbb{R})}^{2}\left(\left\|u_{0}\right\|_{H^{1}(\mathbb{R})}+1\right)^{8 s-2}}{(1-4 s)(4 \pi s)^{4 s}}+\frac{\|\mathcal{K}\|_{L^{2}(\mathbb{R})}^{2}}{b^{2}}\right\}^{\frac{1}{2}}\left\|v_{1}-v_{2}\right\|_{H^{1}(\mathbb{R})} .
\end{aligned}
$$

It can be easily verified using (1.15) that the coefficient of $\left\|v_{1}-v_{2}\right\|_{H^{1}(\mathbb{R})}$ in (2.18) is less than one. This yields that the map $T_{g}: B_{\rho} \rightarrow B_{\rho}$ defined by Eq. (1.11) is a strict contraction for all values of $\varepsilon$ which satisfy inequality (1.15). Its unique fixed point $u_{p}(x)$ is the only solution of problem (1.9) in the ball $B_{\rho}$. By virtue of (2.9) we have that $\left\|u_{p}(x)\right\|_{H^{1}(\mathbb{R})} \rightarrow 0$ as $\varepsilon \rightarrow 0$. The cumulative $u(x) \in H^{1}(\mathbb{R})$ given by (1.8) is a solution of Eq. (1.2). 
We proceed to the establishing of the second main result of our article.

\section{The continuity of the cumulative solution}

Proof of Theorem 5 Apparently, for all the values of $\varepsilon$ which satisfy inequality (1.15), we have

$$
u_{p, 1}=T_{g_{1}} u_{p, 1}, \quad u_{p, 2}=T_{g_{2}} u_{p, 2} .
$$

Hence

$$
u_{p, 1}-u_{p, 2}=T_{g_{1}} u_{p, 1}-T_{g_{1}} u_{p, 2}+T_{g_{1}} u_{p, 2}-T_{g_{2}} u_{p, 2}
$$

such that

$$
\left\|u_{p, 1}-u_{p, 2}\right\|_{H^{1}(\mathbb{R})} \leq\left\|T_{g_{1}} u_{p, 1}-T_{g_{1}} u_{p, 2}\right\|_{H^{1}(\mathbb{R})}+\left\|T_{g_{1}} u_{p, 2}-T_{g_{2}} u_{p, 2}\right\|_{H^{1}(\mathbb{R})} .
$$

Inequality (2.18) gives us

$$
\left\|T_{g_{1}} u_{p, 1}-T_{g_{1}} u_{p, 2}\right\|_{H^{1}(\mathbb{R})} \leq \varepsilon \sigma\left\|u_{p, 1}-u_{p, 2}\right\|_{H^{1}(\mathbb{R})} .
$$

Note that $\varepsilon \sigma \leq \frac{\rho}{\left\|u_{0}\right\|_{H^{1}(\mathbb{R})}+1}<1$ by (1.15) and (1.16) since $\rho \leq 1$ and $u_{0}(x)$ is nontrivial. Hence, we obtain

$$
(1-\varepsilon \sigma)\left\|u_{p, 1}-u_{p, 2}\right\|_{H^{1}(\mathbb{R})} \leq\left\|T_{g_{1}} u_{p, 2}-T_{g_{2}} u_{p, 2}\right\|_{H^{1}(\mathbb{R})} .
$$

According to (3.1), for our fixed point $T_{g_{2}} u_{p, 2}=u_{p, 2}$. Let us introduce $\xi(x):=$ $T_{g_{1}} u_{p, 2}$. Thus, for $0<s<\frac{1}{4}$, we have

$$
\begin{aligned}
& \left(-\frac{d^{2}}{d x^{2}}\right)^{s} \xi(x)-b \frac{d \xi(x)}{d x}=\varepsilon \int_{-\infty}^{\infty} \mathcal{K}(x-y) g_{1}\left(u_{0}(y)+u_{p, 2}(y)\right) d y \\
& \left(-\frac{d^{2}}{d x^{2}}\right)^{s} u_{p, 2}(x)-b \frac{d u_{p, 2}(x)}{d x}=\varepsilon \int_{-\infty}^{\infty} \mathcal{K}(x-y) g_{2}\left(u_{0}(y)+u_{p, 2}(y)\right) d y,
\end{aligned}
$$

Let us designate $G_{1,2}(x):=g_{1}\left(u_{0}(x)+u_{p, 2}(x)\right), G_{2,2}(x):=g_{2}\left(u_{0}(x)+u_{p, 2}(x)\right)$ and apply the standard Fourier transform (2.1) to both sides of problems (3.3) and (3.4) above. This yields

$$
\widehat{\xi}(p)=\varepsilon \sqrt{2 \pi} \frac{\widehat{\mathcal{K}}(p) \widehat{G}_{1,2}(p)}{|p|^{2 s}-i b p}, \quad \widehat{u}_{p, 2}(p)=\varepsilon \sqrt{2 \pi} \frac{\widehat{\mathcal{K}}(p) \widehat{G}_{2,2}(p)}{|p|^{2 s}-i b p} .
$$


Evidently,

$$
\begin{aligned}
& \left\|\xi(x)-u_{p, 2}(x)\right\|_{L^{2}(\mathbb{R})}^{2}=\varepsilon^{2} 2 \pi \int_{-\infty}^{\infty} \frac{|\widehat{\mathcal{K}}(p)|^{2}\left|\widehat{G}_{1,2}(p)-\widehat{G}_{2,2}(p)\right|^{2}}{|p|^{4 s}+b^{2} p^{2}} d p \\
& \quad \leq \varepsilon^{2} 2 \pi \int_{-\infty}^{\infty} \frac{|\widehat{\mathcal{K}}(p)|^{2}\left|\widehat{G}_{1,2}(p)-\widehat{G}_{2,2}(p)\right|^{2}}{|p|^{4 s}} d p .
\end{aligned}
$$

Clearly, the right side of (3.6) can be bounded from above by means of inequality (2.2) as

$$
\begin{aligned}
& \varepsilon^{2} 2 \pi\left[\int_{|p| \leq R} \frac{|\widehat{\mathcal{K}}(p)|^{2}\left|\widehat{G}_{1,2}(p)-\widehat{G}_{2,2}(p)\right|^{2}}{|p|^{4 s}} d p\right. \\
& \left.\quad+\int_{|p|>R} \frac{|\widehat{\mathcal{K}}(p)|^{2}\left|\widehat{G}_{1,2}(p)-\widehat{G}_{2,2}(p)\right|^{2}}{|p|^{4 s}} d p\right] \\
& \quad \leq \varepsilon^{2}\|\mathcal{K}\|_{L^{1}(\mathbb{R})}^{2}\left\{\frac{1}{\pi}\left\|G_{1,2}-G_{2,2}\right\|_{L^{1}(\mathbb{R})}^{2} \frac{R^{1-4 s}}{1-4 s}+\left\|G_{1,2}-G_{2,2}\right\|_{L^{2}(\mathbb{R})}^{2} \frac{1}{R^{4 s}}\right\}
\end{aligned}
$$

with $R \in(0,+\infty)$. We express

$$
G_{1,2}(x)-G_{2,2}(x)=\int_{0}^{u_{0}(x)+u_{p, 2}(x)}\left[g_{1}^{\prime}(z)-g_{2}^{\prime}(z)\right] d z .
$$

Thus

$$
\begin{aligned}
& \left|G_{1,2}(x)-G_{2,2}(x)\right| \leq \max _{z \in I}\left|g_{1}^{\prime}(z)-g_{2}^{\prime}(z)\right|\left|u_{0}(x)+u_{p, 2}(x)\right| \\
& \quad \leq\left\|g_{1}-g_{2}\right\|_{C_{2}(I)}\left|u_{0}(x)+u_{p, 2}(x)\right|
\end{aligned}
$$

so that

$$
\begin{aligned}
& \left\|G_{1,2}-G_{2,2}\right\|_{L^{2}(\mathbb{R})} \leq\left\|g_{1}-g_{2}\right\|_{C_{2}(I)}\left\|u_{0}+u_{p, 2}\right\|_{L^{2}(\mathbb{R})} \\
& \quad \leq\left\|g_{1}-g_{2}\right\|_{C_{2}(I)}\left(\left\|u_{0}\right\|_{H^{1}(\mathbb{R})}+1\right) .
\end{aligned}
$$

Let us use another representation formula, namely

$$
G_{1,2}(x)-G_{2,2}(x)=\int_{0}^{u_{0}(x)+u_{p, 2}(x)} d y\left[\int_{0}^{y}\left(g_{1}^{\prime \prime}(z)-g_{2}^{\prime \prime}(z)\right) d z\right] .
$$

Hence

$$
\begin{aligned}
& \left|G_{1,2}(x)-G_{2,2}(x)\right| \leq \frac{1}{2} \max _{z \in I}\left|g_{1}^{\prime \prime}(z)-g_{2}^{\prime \prime}(z)\right|\left|u_{0}(x)+u_{p, 2}(x)\right|^{2} \\
& \quad \leq \frac{1}{2}\left\|g_{1}-g_{2}\right\|_{C_{2}(I)}\left|u_{0}(x)+u_{p, 2}(x)\right|^{2} .
\end{aligned}
$$


This yields

$$
\begin{aligned}
& \left\|G_{1,2}-G_{2,2}\right\|_{L^{1}(\mathbb{R})} \leq \frac{1}{2}\left\|g_{1}-g_{2}\right\|_{C_{2}(I)}\left\|u_{0}+u_{p, 2}\right\|_{L^{2}(\mathbb{R})}^{2} \\
& \quad \leq \frac{1}{2}\left\|g_{1}-g_{2}\right\|_{C_{2}(I)}\left(\left\|u_{0}\right\|_{H^{1}(\mathbb{R})}+1\right)^{2} .
\end{aligned}
$$

Then we obtain the upper bound for the norm $\left\|\xi(x)-u_{p, 2}(x)\right\|_{L^{2}(\mathbb{R})}^{2}$ given by

$$
\varepsilon^{2}\|\mathcal{K}\|_{L^{1}(\mathbb{R})}^{2}\left(\left\|u_{0}\right\|_{H^{1}(\mathbb{R})}+1\right)^{2}\left\|g_{1}-g_{2}\right\|_{C_{2}(I)}^{2}\left[\frac{1}{4 \pi}\left(\left\|u_{0}\right\|_{H^{1}(\mathbb{R})}+1\right)^{2} \frac{R^{1-4 s}}{1-4 s}+\frac{1}{R^{4 s}}\right] .
$$

This expression can be trivially minimized over $R \in(0,+\infty)$ by virtue of Lemma 4 above. We derive the inequality

$$
\left\|\xi(x)-u_{p, 2}(x)\right\|_{L^{2}(\mathbb{R})}^{2} \leq \varepsilon^{2}\|\mathcal{K}\|_{L^{1}(\mathbb{R})}^{2}\left(\left\|u_{0}\right\|_{H^{1}(\mathbb{R})}+1\right)^{2+8 s} \frac{\left\|g_{1}-g_{2}\right\|_{C_{2}(I)}^{2}}{(1-4 s)(16 \pi s)^{4 s}} .
$$

By means of (3.5) we arrive at

$$
\int_{-\infty}^{\infty} p^{2}\left|\widehat{\xi}(p)-\widehat{u}_{p, 2}(p)\right|^{2} d p \leq \frac{2 \pi \varepsilon^{2}}{b^{2}} \int_{-\infty}^{\infty}|\widehat{\mathcal{K}}(p)|^{2}\left|\widehat{G}_{1,2}(p)-\widehat{G}_{2,2}(p)\right|^{2} d p .
$$

Using inequalities (2.2) and (3.8), the norm $\left\|\frac{d}{d x}\left(\xi(x)-u_{p, 2}(x)\right)\right\|_{L^{2}(\mathbb{R})}^{2}$ can be estimated from above by

$\frac{\varepsilon^{2}}{b^{2}}\|\mathcal{K}\|_{L^{2}(\mathbb{R})}^{2}\left\|G_{1,2}-G_{2,2}\right\|_{L^{1}(\mathbb{R})}^{2} \leq \frac{\varepsilon^{2}}{4 b^{2}}\|\mathcal{K}\|_{L^{2}(\mathbb{R})}^{2}\left(\left\|u_{0}\right\|_{H^{1}(\mathbb{R})}+1\right)^{4}\left\|g_{1}-g_{2}\right\|_{C_{2}(I)}^{2}$.

Thus, $\left\|\xi(x)-u_{p, 2}(x)\right\|_{H^{1}(\mathbb{R})} \leq$

$\leq \varepsilon\left\|g_{1}-g_{2}\right\|_{C_{2}(I)}\left(\left\|u_{0}\right\|_{H^{1}(\mathbb{R})}+1\right)^{2}\left[\frac{\|\mathcal{K}\|_{L^{1}(\mathbb{R})}^{2}\left(\left\|u_{0}\right\|_{H^{1}(\mathbb{R})}+1\right)^{8 s-2}}{(1-4 s)(16 \pi s)^{4 s}}+\frac{\|\mathcal{K}\|_{L^{2}(\mathbb{R})}^{2}}{4 b^{2}}\right]^{\frac{1}{2}}$.

By virtue of inequality (3.2), the norm $\left\|u_{p, 1}-u_{p, 2}\right\|_{H^{1}(\mathbb{R})}$ can be bounded from above by

$$
\begin{aligned}
& \frac{\varepsilon}{1-\varepsilon \sigma}\left(\left\|u_{0}\right\|_{H^{1}(\mathbb{R})}+1\right)^{2} \\
& \quad \times\left[\frac{\|\mathcal{K}\|_{L^{1}(\mathbb{R})}^{2}\left(\left\|u_{0}\right\|_{H^{1}(\mathbb{R})}+1\right)^{8 s-2}}{(1-4 s)(16 \pi s)^{4 s}}+\frac{\|\mathcal{K}\|_{L^{2}(\mathbb{R})}^{2}}{4 b^{2}}\right]^{\frac{1}{2}}\left\|g_{1}-g_{2}\right\|_{C_{2}(I)} .
\end{aligned}
$$

By means of formula (1.17) along with estimate (3.9) inequality (1.18) is valid. 


\section{Auxiliary results}

The solvability conditions for the linear equation with the negative Laplacian raised to a fractional power, the transport term and a square integrable right side

$$
\left(-\frac{d^{2}}{d x^{2}}\right)^{s} u-b \frac{d u}{d x}-a u=f(x), \quad x \in \mathbb{R}, \quad 0<s<1,
$$

where $a \geq 0$ and $b \in \mathbb{R}, b \neq 0$ are constants were derived in the proof of the first theorem of [38]. We will repeat the argument here for the convenience of the readers. Obviously, the operator involved in the left side of (4.1)

$$
\begin{aligned}
& L_{a, b, s}:=\left(-\frac{d^{2}}{d x^{2}}\right)^{s}-b \frac{d}{d x}-a: \quad H^{1}(\mathbb{R}) \rightarrow L^{2}(\mathbb{R}), \quad 0<s \leq \frac{1}{2} \\
& L_{a, b, s}:=\left(-\frac{d^{2}}{d x^{2}}\right)^{s}-b \frac{d}{d x}-a: \quad H^{2 s}(\mathbb{R}) \rightarrow L^{2}(\mathbb{R}), \quad \frac{1}{2}<s<1,
\end{aligned}
$$

is nonselfadjoint. By means of the standard Fourier transform (2.1) it can be easily obtained that the essential spectrum of the operator $L_{a, b, s}$ above is given by

$$
\lambda_{a, b, s}(p):=|p|^{2 s}-a-i b p, \quad p \in \mathbb{R} .
$$

Clearly, in the case when $a>0$, the operator $L_{a, b, s}$ is Fredholm because its essential spectrum does not contain the origin. But when $a$ vanishes, our operator $L_{0, b}, s$ fails to satisfy the Fredholm property since the origin belongs to its essential spectrum. Apparently, in the absense of the drift term, which was discussed for instance in Theorems 1.1 and 1.2 of [37], we deal with the selfadjoint operator

$$
\left(-\frac{d^{2}}{d x^{2}}\right)^{s}-a: \quad H^{2 s}(\mathbb{R}) \rightarrow L^{2}(\mathbb{R}), \quad a>0,
$$

which is non Fredholm. We denote the inner product of two functions as

$$
(f(x), g(x))_{L^{2}(\mathbb{R})}:=\int_{-\infty}^{\infty} f(x) \bar{g}(x) d x,
$$

with a slight abuse of notations when the functions involved in (4.4) are not square integrable. Indeed, if $f(x) \in L^{1}(\mathbb{R})$ and $g(x)$ is bounded, like for instance the functions involved in the inner product in the left side of orthogonality relation (4.5), then the integral in the right side of (4.4) is well defined. We have the following auxiliary proposition.

Lemma 6 Let $f(x): \mathbb{R} \rightarrow \mathbb{R}$ and $f(x) \in L^{2}(\mathbb{R})$, the constant $b \in \mathbb{R}, b \neq 0$. 
(1) If $a>0$ and $0<s \leq \frac{1}{2}$, then problem (4.1) admits a unique solution $u(x) \in$ $H^{1}(\mathbb{R})$.

(2) If $a>0$ and $\frac{1}{2}<s<1$, then Eq. (4.1) has a unique solution $u(x) \in H^{2 s}(\mathbb{R})$.

(3) If $a=0,0<s<\frac{1}{4}$, and, in addition, $f(x) \in L^{1}(\mathbb{R})$, then problem (4.1) possesses a unique solution $u(x) \in H^{1}(\mathbb{R})$.

(4) If $a=0, \frac{1}{4} \leq s \leq \frac{1}{2}$, and, in addition, $x f(x) \in L^{1}(\mathbb{R})$, then Eq. (4.1) admits a unique solution $u(x) \in H^{1}(\mathbb{R})$ if and only if

$$
(f(x), 1)_{L^{2}(\mathbb{R})}=0 .
$$

(5) If $a=0, \frac{1}{2}<s<1$, and, in addition, $x f(x) \in L^{1}(\mathbb{R})$, then problem (4.1) has a unique solution $u(x) \in H^{2 s}(\mathbb{R})$ if and only if orthogonality relation (4.5) holds.

Proof Let us first demonstrate that it would be sufficient to solve our equation in $L^{2}(\mathbb{R})$. Apparently, if $u(x)$ is a square integrable solution of problem (4.1), we have

$$
\left(-\frac{d^{2}}{d x^{2}}\right)^{s} u-b \frac{d u}{d x} \in L^{2}(\mathbb{R})
$$

Then by virtue of the standard Fourier transform (2.1), we obtain

$$
\left(|p|^{2 s}-i b p\right) \widehat{u}(p) \in L^{2}(\mathbb{R}),
$$

that is

$$
\int_{-\infty}^{\infty}\left(|p|^{4 s}+b^{2} p^{2}\right)|\widehat{u}(p)|^{2} d p<\infty
$$

Let $0<s \leq \frac{1}{2}$. Clearly, (4.6) yields

$$
\int_{-\infty}^{\infty} p^{2}|\widehat{u}(p)|^{2} d p<\infty
$$

Thus $\frac{d u}{d x}$ is square integrable on the whole real line and $u(x) \in H^{1}(\mathbb{R})$.

Let $\frac{1}{2}<s<1$. Evidently, (4.6) gives us

$$
\int_{-\infty}^{\infty}|p|^{4 s}|\widehat{u}(p)|^{2} d p<\infty
$$


Hence $\left(-\frac{d^{2}}{d x^{2}}\right)^{s} u \in L^{2}(\mathbb{R})$, such that $u(x) \in H^{2 s}(\mathbb{R})$.

Let us address the uniqueness of a solution to problem (4.1) for $0<s \leq \frac{1}{2}$. When $\frac{1}{2}<s<1$ the argument is similar. Suppose that $u_{1,2}(x) \in H^{1}(\mathbb{R})$ both solve (4.1). Then their difference $w(x):=u_{1}(x)-u_{2}(x) \in H^{1}(\mathbb{R})$ satifies the homogeneous equation

$$
\left(-\frac{d^{2}}{d x^{2}}\right)^{s} w-b \frac{d w}{d x}-a w=0 .
$$

Because the operator $L_{a, b, s}$ defined in (4.2) does not have nontrivial zero modes in $H^{1}(\mathbb{R})$, we obtain that $w(x)=0$ identically on the real line.

By applying the standard Fourier transform (2.1) to both sides of problem (4.1), we arrive at

$$
\widehat{u}(p)=\frac{\widehat{f}(p)}{|p|^{2 s}-a-i b p}, \quad p \in \mathbb{R}, \quad 0<s<1 .
$$

Hence,

$$
\|u\|_{L^{2}(\mathbb{R})}^{2}=\int_{-\infty}^{\infty} \frac{|\widehat{f}(p)|^{2}}{\left(|p|^{2 s}-a\right)^{2}+b^{2} p^{2}} d p .
$$

First we consider assertions (1) and (2) of our lemma. Apparently, (4.8) yields that

$$
\|u\|_{L^{2}(\mathbb{R})}^{2} \leq \frac{1}{C}\|f\|_{L^{2}(\mathbb{R})}^{2}<\infty
$$

as assumed. Here and further down $C$ stands for a finite positive constant. By means of the argument above, when $a>0$, equation (4.1) admits a unique solution $u(x) \in$ $H^{1}(\mathbb{R})$ for $0<s \leq \frac{1}{2}$ and $u(x) \in H^{2 s}(\mathbb{R})$ if $\frac{1}{2}<s<1$.

Then we turn our attention to the situation when $a=0$. Formula (4.7) gives us

$$
\widehat{u}(p)=\frac{\widehat{f}(p)}{|p|^{2 s}-i b p} \chi_{\{|p| \leq 1\}}+\frac{\widehat{f}(p)}{|p|^{2 s}-i b p} \chi_{\{|p|>1\}} .
$$

Here and below, $\chi_{A}$ denotes the characteristic function of a set $A \subseteq \mathbb{R}$. Evidently, the second term in the right side of (4.9) can be bounded from above in the absolute value by

$$
\frac{|\widehat{f}(p)|}{\sqrt{1+b^{2}}} \in L^{2}(\mathbb{R})
$$

since $f(x)$ is square integrable via the one of our assumptions. 
Let $0<s<\frac{1}{4}$. Then, by virtue of (2.2) we arrive at

$$
\left|\frac{\widehat{f}(p)}{|p|^{2 s}-i b p} \chi_{\{|p| \leq 1\}}\right| \leq \frac{|\widehat{f}(p)|}{|p|^{2 s}} \chi_{\{|p| \leq 1\}} \leq \frac{\|f(x)\|_{L^{1}(\mathbb{R})}}{\sqrt{2 \pi}|p|^{2 s}} \chi_{\{|p| \leq 1\}} .
$$

Therefore,

$$
\left\|\frac{\widehat{f}(p)}{|p|^{2 s}-i b p} \chi_{\{|p| \leq 1\}}\right\|_{L^{2}(\mathbb{R})}^{2} \leq \frac{\|f(x)\|_{L^{1}(\mathbb{R})}^{2}}{\pi(1-4 s)}<\infty
$$

because $f(x) \in L^{1}(\mathbb{R})$ as assumed. By means of the argument above, problem (4.1) possesses a unique solution $u(x) \in H^{1}(\mathbb{R})$ in assertion (3) of our lemma.

To establish assertions (4) and (5), we use that

$$
\widehat{f}(p)=\widehat{f}(0)+\int_{0}^{p} \frac{d \widehat{f}(s)}{d s} d s .
$$

Then the first term in the right side of (4.9) can be expressed as

$$
\frac{\widehat{f}(0)}{|p|^{2 s}-i b p} \chi_{\{|p| \leq 1\}}+\frac{\int_{0}^{p} \frac{d \widehat{f}(s)}{d s} d s}{|p|^{2 s}-i b p} \chi_{\{|p| \leq 1\}} .
$$

Definition (2.1) of the standard Fourier transform gives us

$$
\left|\frac{d \widehat{f}(p)}{d p}\right| \leq \frac{1}{\sqrt{2 \pi}}\|x f(x)\|_{L^{1}(\mathbb{R})} .
$$

This allows us to obtain the upper bound in the absolute value on the second term in (4.10) as

$$
\frac{1}{\sqrt{2 \pi}} \frac{\|x f(x)\|_{L^{1}(\mathbb{R})}}{|b|} \chi_{\{|p| \leq 1\}} \in L^{2}(\mathbb{R})
$$

via the assumptions of the lemma. We analyze the first term in (4.10) given by

$$
\frac{\widehat{f}(0)}{|p|^{2 s}-i b p} \chi\{|p| \leq 1\}
$$

Obviously, when $\frac{1}{4} \leq s \leq \frac{1}{2}$, expression (4.11) can be easily estimated from below in the absolute value by

$$
\frac{|\widehat{f}(0)|}{|p|^{2 s} \sqrt{1+b^{2}}} \chi_{\{|p| \leq 1\}},
$$


which does not belong to $L^{2}(\mathbb{R})$ unless $\widehat{f}(0)=0$. This implies orthogonality condition (4.5). In case (4), the square integrability of the solution $u(x)$ to problem (4.1) is equivalent to $u(x) \in H^{1}(\mathbb{R})$.

Evidently, for $\frac{1}{2}<s<1$ expression (4.11) can be trivially bounded below in the absolute value by

$$
\frac{|\widehat{f}(0)|}{|p| \sqrt{1+b^{2}}} \chi_{\{|p| \leq 1\}}
$$

which is not square integrable on the whole real line unless orthogonality relation (4.5) holds. In case (5), the square integrability of the solution $u(x)$ to Eq. (4.1) is equivalent to $u(x) \in H^{2 s}(\mathbb{R})$.

Note that in the situation when $a=0$ and $0<s<\frac{1}{4}$ of the lemma above the orthogonality conditions are not needed as distinct from assertions (4) and (5). In case (3) under our assumptions Eq. (4.1) admits a unique solution $u_{0}(x) \in H^{1}(\mathbb{R})$ which we use in the proofs of the main results of the article.

Related to Eq. (4.1) on the real line, we consider the sequence of approximate equations with $m \in \mathbb{N}$ given by

$$
\left(-\frac{d^{2}}{d x^{2}}\right)^{s} u_{m}-b \frac{d u_{m}}{d x}-a u_{m}=f_{m}(x), \quad x \in \mathbb{R}, \quad 0<s<1,
$$

where $a \geq 0$ and $b \in \mathbb{R}, b \neq 0$ are constants and the right side of (4.12) converges to the right side of (4.1) in $L^{2}(\mathbb{R})$ as $m \rightarrow \infty$. We will prove that, for $0<s \leq \frac{1}{2}$, under the certain technical assumptions, each of problems (4.12) admits a unique solution $u_{m}(x) \in H^{1}(\mathbb{R})$, limiting equation (4.1) has a unique solution $u(x) \in H^{1}(\mathbb{R})$, and $u_{m}(x) \rightarrow u(x)$ in $H^{1}(\mathbb{R})$ as $m \rightarrow \infty$, which is the so-called existence of solutions in the sense of sequences (see [24,37,38] and the references therein). When $\frac{1}{2}<s<1$, the similar ideas will be exploited in $H^{2 s}(\mathbb{R})$. Our final proposition is as follows.

Lemma 7 Let the constant $b \in \mathbb{R}, b \neq 0, m \in \mathbb{N}, f_{m}(x): \mathbb{R} \rightarrow \mathbb{R}$ and $f_{m}(x) \in$ $L^{2}(\mathbb{R})$. Furthermore, $f_{m}(x) \rightarrow f(x)$ in $L^{2}(\mathbb{R})$ as $m \rightarrow \infty$.

(1) If $a>0$ and $0<s \leq \frac{1}{2}$, then problems (4.1) and (4.12) admit unique solutions $u(x) \in H^{1}(\mathbb{R})$ and $u_{m}(x) \in H^{1}(\mathbb{R})$ respectively, such that $u_{m}(x) \rightarrow u(x)$ in $H^{1}(\mathbb{R})$ as $m \rightarrow \infty$.

(2) If $a>0$ and $\frac{1}{2}<s<1$, then Eqs. (4.1) and (4.12) have unique solutions $u(x) \in$ $H^{2 s}(\mathbb{R})$ and $u_{m}(x) \in H^{2 s}(\mathbb{R})$ respectively, such that $u_{m}(x) \rightarrow u(x)$ in $H^{2 s}(\mathbb{R})$ as $m \rightarrow \infty$.

(3) If $a=0$ and $0<s<\frac{1}{4}$, and in addition $f_{m}(x) \in L^{1}(\mathbb{R})$ and $f_{m}(x) \rightarrow f(x)$ in $L^{1}(\mathbb{R})$ as $m \rightarrow \infty$, then problems (4.1) and (4.12) possess unique solutions 
$u(x) \in H^{1}(\mathbb{R})$ and $u_{m}(x) \in H^{1}(\mathbb{R})$ respectively, such that $u_{m}(x) \rightarrow u(x)$ in $H^{1}(\mathbb{R})$ as $m \rightarrow \infty$.

(4) If $a=0$ and $\frac{1}{4} \leq s \leq \frac{1}{2}$, let in addition $x f_{m}(x) \in L^{1}(\mathbb{R})$ and $x f_{m}(x) \rightarrow x f(x)$ in $L^{1}(\mathbb{R})$ as $m \rightarrow \infty$. Moreover,

$$
\left(f_{m}(x), 1\right)_{L^{2}(\mathbb{R})}=0, \quad m \in \mathbb{N}
$$

holds. Then Eqs. (4.1) and (4.12) admit unique solutions $u(x) \in H^{1}(\mathbb{R})$ and $u_{m}(x) \in H^{1}(\mathbb{R})$ respectively, such that $u_{m}(x) \rightarrow u(x)$ in $H^{1}(\mathbb{R})$ as $m \rightarrow \infty$.

(5) If $a=0$ and $\frac{1}{2}<s<1$, let in addition $x f_{m}(x) \in L^{1}(\mathbb{R})$ and $x f_{m}(x) \rightarrow x f(x)$ in $L^{1}(\mathbb{R})$ as $m \rightarrow \infty$. Furthermore, orthogonality relations (4.13) hold. Then problems (4.1) and (4.12) have unique solutions $u(x) \in H^{2 s}(\mathbb{R})$ and $u_{m}(x) \in$ $H^{2 s}(\mathbb{R})$ respectively, such that $u_{m}(x) \rightarrow u(x)$ in $H^{2 s}(\mathbb{R})$ as $m \rightarrow \infty$.

Proof Let us assume that problems (4.1) and (4.12) admit unique solutions $u(x) \in$ $H^{1}(\mathbb{R})$ and $u_{m}(x) \in H^{1}(\mathbb{R}), m \in \mathbb{N}$ respectively for $0<s \leq \frac{1}{2}$, and analogously $u(x) \in H^{2 s}(\mathbb{R})$ and $u_{m}(x) \in H^{2 s}(\mathbb{R}), m \in \mathbb{N}$ if $\frac{1}{2}<s<1$, such that $u_{m}(x) \rightarrow u(x)$ in $L^{2}(\mathbb{R})$ as $m \rightarrow \infty$. Then $u_{m}(x)$ also tends to $u(x)$ in $H^{1}(\mathbb{R})$ as $m \rightarrow \infty$ if $0<s \leq \frac{1}{2}$, and analogously $u_{m}(x) \rightarrow u(x)$ in $H^{2 s}(\mathbb{R})$ as $m \rightarrow \infty$ for $\frac{1}{2}<s<1$. Indeed, Eqs. (4.1) and (4.12) give us

$$
\begin{aligned}
& \left\|\left(-\frac{d^{2}}{d x^{2}}\right)^{s}\left(u_{m}-u\right)-b \frac{d\left(u_{m}-u\right)}{d x}\right\|_{L^{2}(\mathbb{R})} \leq \\
& \leq\left\|f_{m}-f\right\|_{L^{2}(\mathbb{R})}+a\left\|u_{m}-u\right\|_{L^{2}(\mathbb{R})} .
\end{aligned}
$$

Clearly, the right side of inequality (4.14) converges to zero as $m \rightarrow \infty$ due to our assumptions above. By virtue of the standard Fourier transform (2.1), we easily derive

$$
\int_{-\infty}^{\infty}\left(|p|^{4 s}+b^{2} p^{2}\right)\left|\widehat{u}_{m}(p)-\widehat{u}(p)\right|^{2} d p \rightarrow 0, \quad m \rightarrow \infty .
$$

Let $0<s \leq \frac{1}{2}$. By means of (4.15),

$$
\int_{-\infty}^{\infty} p^{2}\left|\widehat{u}_{m}(p)-\widehat{u}(p)\right|^{2} d p \rightarrow 0, \quad m \rightarrow \infty
$$

such that

$$
\frac{d u_{m}}{d x} \rightarrow \frac{d u}{d x} \quad \text { in } \quad L^{2}(\mathbb{R}), \quad m \rightarrow \infty
$$


Hence, when $0<s \leq \frac{1}{2}$, norm definition (1.6) implies that $u_{m}(x) \rightarrow u(x)$ in $H^{1}(\mathbb{R})$ as $m \rightarrow \infty$.

Suppose that $\frac{1}{2}<s<1$. By virtue of (4.15),

$$
\int_{-\infty}^{\infty}|p|^{4 s}\left|\widehat{u}_{m}(p)-\widehat{u}(p)\right|^{2} d p \rightarrow 0, \quad m \rightarrow \infty
$$

so that

$$
\left(-\frac{d^{2}}{d x^{2}}\right)^{s} u_{m} \rightarrow\left(-\frac{d^{2}}{d x^{2}}\right)^{s} u \text { in } \quad L^{2}(\mathbb{R}), \quad m \rightarrow \infty
$$

Thus, if $\frac{1}{2}<s<1$, norm definition (1.5) yields that $u_{m}(x) \rightarrow u(x)$ in $H^{2 s}(\mathbb{R})$ as $m \rightarrow \infty$. $^{2}$

Let us apply the standard Fourier transform (2.1) to both sides of Eq. (4.12). This yields

$$
\widehat{u}_{m}(p)=\frac{\widehat{f_{m}}(p)}{|p|^{2 s}-a-i b p}, \quad m \in \mathbb{N}, \quad p \in \mathbb{R}, \quad 0<s<1
$$

Let us discuss assertions (1) and (2). By means of parts (1) and (2) of Lemma 6 above, for $a>0$, problems (4.1) and (4.12) admit unique solutions $u(x) \in H^{1}(\mathbb{R})$ and $u_{m}(x) \in H^{1}(\mathbb{R}), m \in \mathbb{N}$ respectively if $0<s \leq \frac{1}{2}$ and analogously $u(x) \in H^{2 s}(\mathbb{R})$ and $u_{m}(x) \in H^{2 s}(\mathbb{R}), m \in \mathbb{N}$ provided that $\frac{1}{2}<s<1$. By virtue of (4.16) along with (4.7), we arrive at

$$
\left\|u_{m}-u\right\|_{L^{2}(\mathbb{R})}^{2}=\int_{-\infty}^{\infty} \frac{\left|\widehat{f}_{m}(p)-\widehat{f}(p)\right|^{2}}{\left(|p|^{2 s}-a\right)^{2}+b^{2} p^{2}} d p .
$$

Therefore

$$
\left\|u_{m}-u\right\|_{L^{2}(\mathbb{R})} \leq \frac{1}{C}\left\|f_{m}-f\right\|_{L^{2}(\mathbb{R})} \rightarrow 0, \quad m \rightarrow \infty
$$

as assumed. Hence, for $a>0$, we have $u_{m}(x) \rightarrow u(x)$ in $H^{1}(\mathbb{R})$ as $m \rightarrow \infty$ if $0<s \leq \frac{1}{2}$ and $u_{m}(x) \rightarrow u(x)$ in $H^{2 s}(\mathbb{R})$ as $m \rightarrow \infty$ when $\frac{1}{2}<s<1$ due to the above argument.

Let us complete the proof by studying the case of $a=0$. According to the part (a) of Lemma 3.3 of [27], under the given conditions

$$
(f(x), 1)_{L^{2}(\mathbb{R})}=0
$$


in assertions (4) and (5) of our lemma. By means of the results of parts (3)-(5) of Lemma 6 above, problems (4.1) and (4.12) with $a=0$ possess unique solutions $u(x) \in$ $H^{1}(\mathbb{R})$ and $u_{m}(x) \in H^{1}(\mathbb{R}), m \in \mathbb{N}$ respectively for $0<s \leq \frac{1}{2}$ and analogously $u(x) \in H^{2 s}(\mathbb{R})$ and $u_{m}(x) \in H^{2 s}(\mathbb{R}), m \in \mathbb{N}$ when $\frac{1}{2}<s<1$. Formulas (4.16) and (4.7) give us

$$
\widehat{u}_{m}(p)-\widehat{u}(p)=\frac{\widehat{f}_{m}(p)-\widehat{f}(p)}{|p|^{2 s}-i b p} \chi_{\{|p| \leq 1\}}+\frac{\widehat{f}_{m}(p)-\widehat{f}(p)}{|p|^{2 s}-i b p} \chi_{\{|p|>1\}} .
$$

Evidently, the second term in the right side of (4.18) can be estimated from above in the $L^{2}(\mathbb{R})$ norm by

$$
\frac{1}{\sqrt{1+b^{2}}}\left\|f_{m}-f\right\|_{L^{2}(\mathbb{R})} \rightarrow 0, \quad m \rightarrow \infty
$$

via the one of our assumptions. Suppose $0<s<\frac{1}{4}$. Let us use an analog of inequality (2.2) to derive

$$
\left|\frac{\widehat{f_{m}}(p)-\widehat{f}(p)}{|p|^{2 s}-i b p} \chi_{\{|p| \leq 1\}}\right| \leq \frac{\left|\widehat{f}_{m}(p)-\widehat{f}(p)\right|}{|p|^{2 s}} \chi_{\{|p| \leq 1\}} \leq \frac{\left\|f_{m}-f\right\|_{L^{1}(\mathbb{R})}}{\sqrt{2 \pi}|p|^{2 s}} \chi_{\{|p| \leq 1\}}
$$

Hence

$$
\left\|\frac{\widehat{f_{m}}(p)-\widehat{f}(p)}{|p|^{2 s}-i b p} \chi_{\{|p| \leq 1\}}\right\|_{L^{2}(\mathbb{R})} \leq \frac{\left\|f_{m}-f\right\|_{L^{1}(\mathbb{R})}}{\sqrt{\pi(1-4 s)}} \rightarrow 0, \quad m \rightarrow \infty
$$

due to the one of the assumptions of the lemma. By virtue of the argument above, we obtain that $u_{m}(x) \rightarrow u(x)$ in $H^{1}(\mathbb{R})$ as $m \rightarrow \infty$ in the situation when $a=0$ and $0<s<\frac{1}{4}$.

Let us use orthogonality conditions (4.17) and (4.13) to establish assertions (4) and (5). By virtue of definition (2.1) of the standard Fourier transform, we obtain

$$
\widehat{f}(0)=0, \quad \widehat{f_{m}}(0)=0, \quad m \in \mathbb{N} .
$$

This yields

$$
\widehat{f}(p)=\int_{0}^{p} \frac{d \widehat{f}(s)}{d s} d s, \quad \widehat{f_{m}}(p)=\int_{0}^{p} \frac{d \widehat{f}_{m}(s)}{d s} d s, \quad m \in \mathbb{N} .
$$


Therefore, the first term in the right side of (4.18) in assertions (4) and (5) of our lemma is given by

$$
\frac{\int_{0}^{p}\left[\frac{d \widehat{f}_{m}(s)}{d s}-\frac{d \widehat{f}(s)}{d s}\right] d s}{|p|^{2 s}-i b p} \chi_{\{|p| \leq 1\}} .
$$

It easily follows from definition (2.1) of the standard Fourier transform that

$$
\left|\frac{d \widehat{f}_{m}(p)}{d p}-\frac{d \widehat{f}(p)}{d p}\right| \leq \frac{1}{\sqrt{2 \pi}}\left\|x f_{m}(x)-x f(x)\right\|_{L^{1}(\mathbb{R})} .
$$

Therefore,

$$
\left|\frac{\int_{0}^{p}\left[\frac{d \widehat{f}_{m}(s)}{d s}-\frac{d \widehat{f}(s)}{d s}\right] d s}{|p|^{2 s}-i b p} \chi_{\{|p| \leq 1\}}\right| \leq \frac{\left\|x f_{m}(x)-x f(x)\right\|_{L^{1}(\mathbb{R})}}{\sqrt{2 \pi}|b|} \chi_{\{|p| \leq 1\}},
$$

such that

$$
\left\|\frac{\int_{0}^{p}\left[\frac{d \widehat{f}_{m}(s)}{d s}-\frac{d \widehat{f}(s)}{d s}\right] d s}{|p|^{2 s}-i b p} \chi_{\{|p| \leq 1\}}\right\|_{L^{2}(\mathbb{R})} \leq \frac{\left\|x f_{m}(x)-x f(x)\right\|_{L^{1}(\mathbb{R})}}{\sqrt{\pi}|b|} \rightarrow 0
$$

as $m \rightarrow \infty$ as assumed. Thus, $u_{m}(x) \rightarrow u(x)$ in $L^{2}(\mathbb{R})$ as $m \rightarrow \infty$. Arguing as above in the case when $a=0$, we observe that $u_{m}(x) \rightarrow u(x)$ in $H^{1}(\mathbb{R})$ as $m \rightarrow \infty$ for $\frac{1}{4} \leq s \leq \frac{1}{2}$ and $u_{m}(x) \rightarrow u(x)$ in $H^{2 s}(\mathbb{R})$ as $m \rightarrow \infty$ if $\frac{1}{2}<s<1$.

Acknowledgements This work is supported by the Ministry of Science and Higher Education of the Russian Federation: agreement no.075-03-2020-223/3 (FSSF-2020-0018).

Funding Funding was provided by Canadian Network for Research and Innovation in Machining Technology, Natural Sciences and Engineering Research Council of Canada (Grant No. NA 7901)

Data availibility All data generated or analyzed during this study are included in this published article.

\section{Declarations}

Conflict of interest There has been no any conflict of interest during the work on this manuscript.

\section{References}

1. Alfimov, G.L., Medvedeva, E.V., Pelinovsky, D.E.: Wave systems with an infinite number of localized traveling waves. Phys. Rev. Lett. 112, 054103 (2014)

2. Alfimov, G.L., Korobeinikov, A.S., Lustri, C.J., Pelinovsky, D.E.: Standing lattice solitons in the discrete NLS equation with saturation. Nonlinearity 32(9), 3445-3484 (2019)

3. Amrouche, C., Girault, V., Giroire, J.: Dirichlet and Neumann exterior problems for the $n$-dimensional Laplace operator: an approach in weighted Sobolev spaces. J. Math. Pures Appl. 76(1), 55-81 (1997) 
4. Amrouche, C., Bonzom, F.: Mixed exterior Laplace's problem. J. Math. Anal. Appl. 338, 124-140 (2008)

5. Bolley, P., Pham, T.L.: Propriété d'indice en théorie Holderienne pour des opérateurs différentiels elliptiques dans $R^{n}$. J. Math. Pures Appl. 72(1), 105-119 (1993)

6. Bolley, P., Pham, T.L.: Propriété d'indice en théorie Hölderienne pour le problème extérieur de Dirichlet. Commun. Partial Differ. Equ. 26(1-2), 315-334 (2001)

7. Benkirane, N.: Propriété d'indice en théorie Holderienne pour des opérateurs elliptiques dans $R^{n}$. CRAS 577-307(Série I), 577-580 (1988)

8. Carreras, B., Lynch, V., Zaslavsky, G.: Anomalous diffusion and exit time distribution of particle tracers in plasma turbulence model. Phys. Plasmas 8, 5096-5103 (2001)

9. Cuccagna, S., Pelinovsky, D., Vougalter, V.: Spectra of positive and negative energies in the linearized NLS problem. Commun. Pure Appl. Math. 58(1), 1-29 (2005)

10. Ducrot, A., Marion, M., Volpert, V.: Systemes de réaction-diffusion sans propriété de Fredholm. CRAS 340, 659-664 (2005)

11. Ducrot, A., Marion, M., Volpert, V.: Reaction-diffusion problems with non Fredholm operators. Adv. Diff. Equ. 13(11-12), 1151-1192 (2008)

12. Efendiev, M.: Fredholm Structures, Topological Invariants and Applications. AIMS Series on Differential Equations \& Dynamical Systems, vol. 3. American Institute of Mathematical Sciences (AIMS), Springfield (2009)

13. Efendiev, M., Vougalter, V.: Solvability of some integro-differential equations with drift. Osaka J. Math. 57(2), 247-265 (2020)

14. Efendiev, M., Vougalter, V.: Solvability in the sense of sequences for some fourth order non-Fredholm operators. J. Differ. Equ. 271, 280-300 (2021)

15. Gebran, H.G., Stuart, C.A.: Fredholm and properness properties of quasilinear elliptic systems of second order. Proc. Edinb. Math. Soc. (2) 48(1), 91-124 (2005)

16. Lieb, E., Loss, M.: Analysis. Graduate Studies in Mathematics, vol. 14, American Mathematical Society, Providence (1997)

17. Manandhar, P., Jang, J., Schatz, G.C., Ratner, M.A., Hong, S.: Anomalous surface diffusion in nanoscale direct deposition processes. Phys. Rev. Lett. 90, 4043-4052 (2003)

18. Maz'ja, V.G.: Weak solutions of the Dirichlet and Neumann problems. Trudy Moskov. Mat. Obsc. 20, 137-172 (1969). (Russian)

19. Maz'ja, V.G., Otelbaev, M.: Imbedding theorems and the spectrum of a certain pseudodifferential operator. Sibirsk. Mat. Z. 18(5), 1073-1087 (1977). (Russian)

20. Metzler, R., Klafter, J.: The random walk's guide to anomalous diffusion: a fractional dynamics approach. Phys. Rep. 339, 1-77 (2000)

21. Sancho, J., Lacasta, A., Lindenberg, K., Sokolov, I., Romero, A.: Diffusion on a solid surface: anomalous is normal. Phys. Rev. Lett. 92, 250601 (2004)

22. Scher, H., Montroll, E.: Anomalous transit-time dispersion in amorphous solids. Phys. Rev. B 12, 2455-2477 (1975)

23. Solomon, T., Weeks, E., Swinney, H.: Observation of anomalous diffusion and Lévy flights in a twodimensional rotating flow. Phys. Rev. Lett. 71, 3975-3978 (1993)

24. Volpert, V.: Elliptic Partial Differential Equations. Volume 1: Fredholm Theory of Elliptic Problems in Unbounded Domains. Monographs in Mathematics, vol. 101. Birkhäuser/Springer Basel AG, Basel (2011)

25. Volpert, V., Vougalter, V.: On the solvability conditions for a linearized Cahn-Hilliard equation. Rend. Istit. Mat. Univ. Trieste 43, 1-9 (2011)

26. Volpert, V., Vougalter, V.: Emergence and propagation of patterns in nonlocal reaction-diffusion equations arising in the theory of speciation. In: Dispersal, Individual Movement and Spatial Ecology, Lecture Notes in Math., 2071, pp. 331-353. Springer, Heidelberg (2013)

27. Volpert, V., Vougalter, V.: Solvability in the sense of sequences to some non-Fredholm operators. Electron. J. Differ. Equ. 160, 1-16 (2013)

28. Volpert, V.A., Nec, Y., Nepomnyashchy, A.A.: Exact solutions in front propagation problems with superdiffusion. Phys. D 239(3-4), 134-144 (2010)

29. Volpert, V.A., Nec, Y., Nepomnyashchy, A.A.: Fronts in anomalous diffusion-reaction systems. Philos. Trans. R. Soc. Lond. Ser. A Math. Phys. Eng. Sci. 371(1982), 20120179 (2013)

30. Vougalter, V.: On threshold eigenvalues and resonances for the linearized NLS equation. Math. Model. Nat. Phenom. 5(4), 448-469 (2010) 
31. Vougalter, V., Volpert, V.: Solvability conditions for some non-Fredholm operators. Proc. Edinb. Math. Soc. (2) 54(1), 249-271 (2011)

32. Vougalter, V., Volpert, V.: On the existence of stationary solutions for some non-Fredholm integrodifferential equations. Doc. Math. 16, 561-580 (2011)

33. Vougalter, V., Volpert, V.: On the solvability conditions for the diffusion equation with convection terms. Commun. Pure Appl. Anal. 11(1), 365-373 (2012)

34. Vougalter, V., Volpert, V.: Solvability conditions for a linearized Cahn-Hilliard equation of sixth order. Math. Model. Nat. Phenom. 7(2), 146-154 (2012)

35. Vougalter, V., Volpert, V.: Solvability conditions for some linear and nonlinear non-Fredholm elliptic problems. Anal. Math. Phys. 2(4), 473-496 (2012)

36. Vougalter, V., Volpert, V.: Solvability of some integro-differential equations with anomalous diffusion. Regularity and stochasticity of nonlinear dynamical systems. Nonlinear Syst. Complex. 21, 1-17 (2018)

37. Vougalter, V., Volpert, V.: On the solvability in the sense of sequences for some non-Fredholm operators related to the anomalous diffusion. In: Analysis of Pseudo-Differential Operators, Trends Math., pp. 229-257. Birkhäuser/Springer, Cham (2019)

38. Vougalter, V.: On solvability in the sense of sequences for some non-Fredholm operators with drift and anomalous diffusion. J. Math. Sci. (N.Y.) 250(2), 285-299 (2020)

Publisher's Note Springer Nature remains neutral with regard to jurisdictional claims in published maps and institutional affiliations. 